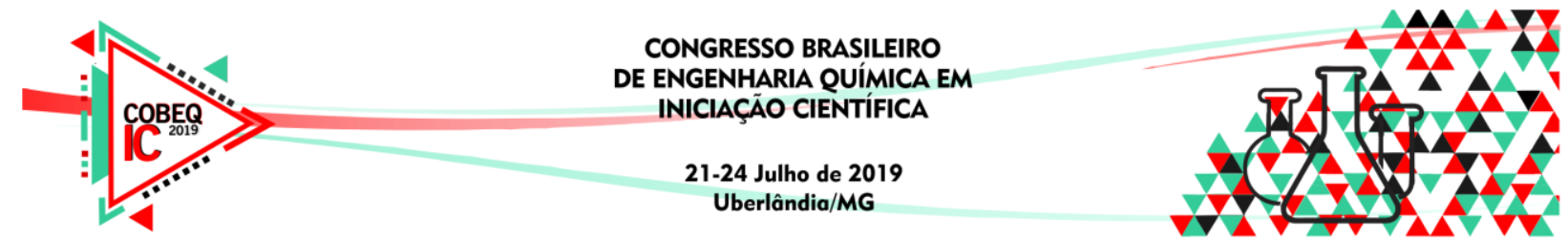

\title{
OXIDAÇÃO EM ÁGUA SUPERCRÍTICA: INFLUÊNCIA DA TEMPERATURA NA DEGRADAÇÃO DE MATÉRIA ORGÂNICA E AMÔNIA DE LIXIVIADO DE ATERRO SANITÁRIO
}

\author{
R. S. SYLLOS ${ }^{1}$, A. P. J. SCANDELAI ${ }^{1}$, D. C. C. MARTINS ${ }^{1}$, L. CARDOZO \\ FILHO $^{1}$ e C. R. G. TAVARES ${ }^{1}$ \\ ${ }^{1}$ Universidade Estadual de Maringá, Departamento de Engenharia Química \\ E-mail para contato: renansyllos@ outlook.com
}

\begin{abstract}
RESUMO - Os lixiviados gerados em aterros sanitários apresentam composição variada e complexa, o que dificulta o seu tratamento por um único processo. A técnica de oxidação em água supercrítica (OASc) tem sido eficiente para a degradação dos poluentes desse efluente; entretanto, tem sido verificada a formação de nitrogênio amoniacal total $\left(\mathrm{N}-\mathrm{NH}_{3}\right)$ após esse processo. Diante disso, este estudo objetivou avaliar a influência da temperatura de $\mathrm{OASc}$ na remoção de $\mathrm{N}^{-\mathrm{NH}_{3}}$ do lixiviado pré-tratado do aterro sanitário de Maringá-PR. A OASc foi realizada com pressão de $23 \mathrm{MPa}$, temperaturas de 600 e $700{ }^{\circ} \mathrm{C}$ e tempos espaciais de 31 e $32 \mathrm{~s}$, respectivamente. A OASc, em ambas as temperaturas, se apresentou eficiente na despoluição do lixiviado, no entanto, não foi capaz de remover completamente o $\mathrm{N}-\mathrm{NH}_{3}$, embora o aumento de temperatura tenha favorecido a oxidação de compostos nitrogenados. Dessa forma, condições ainda mais severas de operação seriam necessárias para promover a completa remoção desse composto recalcitrante. Alternativamente, processos específicos poderiam ser adicionados sequencialmente a OASc como, por exemplo, a adsorção.
\end{abstract}

\section{INTRODUÇÃO}

No processo da decomposição dos resíduos sólidos nos aterros sanitários ocorre a geração de subprodutos em estado gasoso (biogás) e líquido (lixiviado). O lixiviado é um efluente com composição físico-química e microbiológica bastante complexa e variável, possuindo poluentes de difícil remoção (Kjeldsen et al., 2002). Essas características evidenciam a sua necessidade de ser tratado antes do lançamento em corpos receptores.

Devido a essa complexidade e à baixa eficiência de processos únicos ou convencionais para sua degradação, alternativas avançadas de tratamento, combinadas ou não com outras técnicas, têm sido investigadas, como a oxidação em água supercrítica (OASc). Devido às altas taxas de transferências de massa e energia que ocorrem nas condições supercríticas de pressão e temperatura da água, isto é, $22,1 \mathrm{MPa}$ e $374,1{ }^{\circ} \mathrm{C}$, a OASc apresenta elevada eficiência de remoção de compostos orgânicos de efluentes diversos, incluindo lixiviados de aterros sanitários, em curtos tempos reacionais (Weijin e Xuejun, 2010).

A formação de nitrogênio amoniacal total $\left(\mathrm{N}-\mathrm{NH}_{3}\right)$ durante a degradação de lixiviado pelo processo supercrítico foi recentemente verificada por nosso grupo de pesquisa (Scandelai 


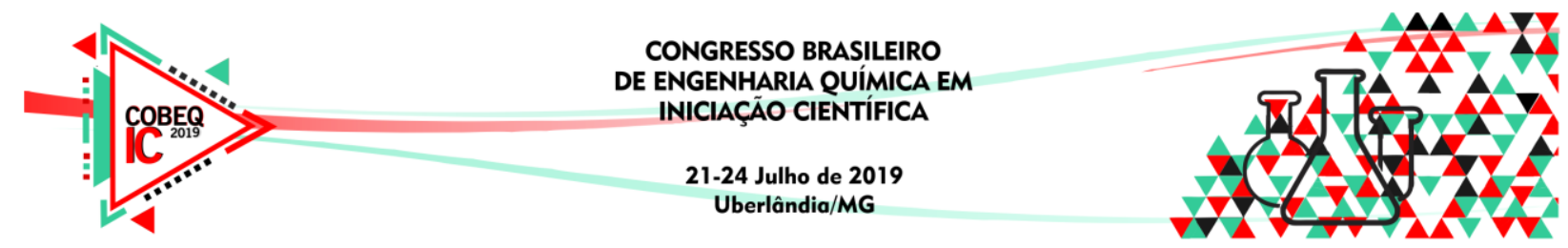

et al., 2018) e, diante disso, este estudo objetivou verificar a influência da temperatura supercrítica na remoção de $\mathrm{N}-\mathrm{NH}_{3}$ do lixiviado pré-tratado do aterro sanitário de Maringá-PR.

\section{METODOLOGIA}

\subsection{Coleta do Lixiviado}

O lixiviado gerado no aterro sanitário de Maringá-PR é tratado no próprio aterro pelas técnicas convencionais de coagulação-floculação-sedimentação, degradação biológica e decantação. O efluente resultante desse processo (lixiviado pré-tratado - LPT) foi coletado na lagoa de decantação da estação de tratamento do local e armazenado a $4{ }^{\circ} \mathrm{C}$ até a sua caracterização e realização dos ensaios experimentais, conforme recomendado por APHA/AWWA/WEF (1998).

\subsection{Tratamento do Lixiviado}

Após caracterização físico-química, o LPT foi filtrado em papel quantitativo faixa branca JP40, para remoção de impurezas grosseiras que pudessem comprometer o sistema supercrítico. Posteriormente, foi submetido à degradação por OASc (Figura 1). O processo se iniciava pela sucção do LPT, contido em um reservatório (1), por uma bomba de alta pressão (Thar Process, P50) a 5,0 $\mathrm{g} \mathrm{min}^{-1}$ (2), sendo enviado ao pré-aquecedor (mantido a $250{ }^{\circ} \mathrm{C}$ ) (3) e ao reator supercrítico (4), os quais eram envoltos por fornos bipartidos (5). Posteriormente, o fluido seguia para o trocador de calor (6) e para o condensador (7), o qual era refrigerado por um banho termostatizado mantido a $10{ }^{\circ} \mathrm{C}$, permitindo o retorno do fluido ao estado líquido. $\mathrm{O}$ efluente tratado era coletado ao final do sistema (8) e analisado físicoquimicamente. As temperaturas do pré-aquecedor e do reator supercrítico eram controladas por meio de termopares (T1 e T2). A pressão do sistema era ajustada por uma válvula de contrapressão (V1) e monitorada por um manômetro (P1). Detalhes do sistema de OASc foram previamente descritos por Scandelai et al. (2018).

Figura 1 - Aparato experimental do processo de OASc. Adaptado de Scandelai et al. (2017).

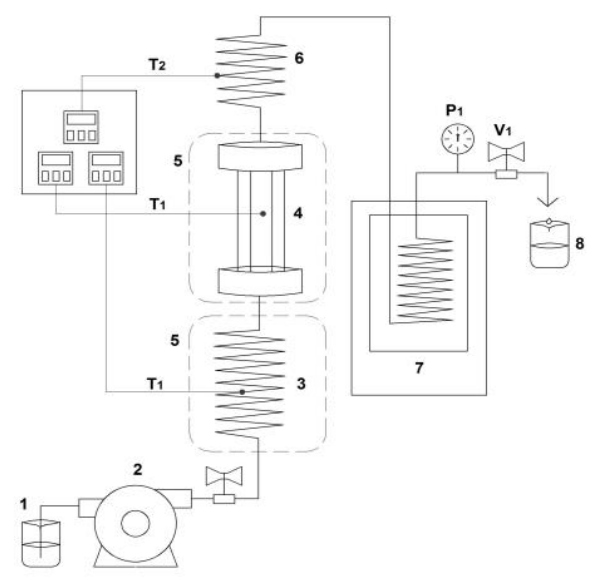

As temperaturas do reator supercrítico foram mantidas em 600 ou $700{ }^{\circ} \mathrm{C}$, a fim de avaliar a sua influência na remoção de matéria orgânica e amônia. A pressão foi mantida em 


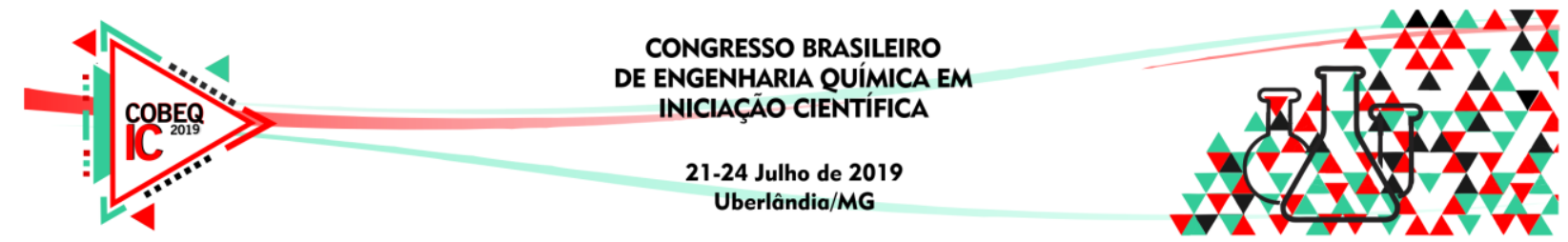

$23 \mathrm{MPa}$ e o tempo espacial $(\tau)$ foi calculado utilizando a Equação 1, adaptada de Van Bennekom et al. (2011), considerando: o volume geométrico do reator supercrítico (Vr, em $\left.\mathrm{cm}^{3}\right)$; a densidade do fluido na temperatura e pressão de operação $\left(\rho_{\mathrm{T}, \mathrm{P}}\right.$, em $\left.\mathrm{g} \mathrm{cm}^{-3}\right)$, obtida pelo National Institute of Standards and Technology (2018); o fluxo mássico de alimentação em temperatura e pressão ambiente, $25{ }^{\circ} \mathrm{C}$ e $101,325 \mathrm{kPa}\left(\varphi_{\mathrm{m}, \mathrm{SATP}}\right.$, em $\left.\mathrm{g} \mathrm{min}{ }^{-1}\right)$; e a porosidade do leito do reator $(\varepsilon)$.

$$
\tau=\frac{V_{r} \times \rho_{T, p} \times \varepsilon}{\varphi_{m, S A T P}}
$$

Para cada experimento, a vazão mássica $\left(\varphi_{\mathrm{m}}\right)$ era medida a cada $30 \mathrm{~min}$, com o auxílio de uma balança analítica (Bel Engineering, L-12001) e obtidas utilizando a menor vazão permitida pela bomba de alta pressão. Na OASc, a $600{ }^{\circ} \mathrm{C}$ e $700{ }^{\circ} \mathrm{C}$, as $\varphi_{\mathrm{m}}$ foram, respectivamente, de 4,65 $\pm 0,05 \mathrm{~g} \mathrm{~min}^{-1}$ e 4,49 $\pm 0,14 \mathrm{~g} \mathrm{~min}^{-1}$ (média \pm desvio), com $\tau$ de $31 \mathrm{e}$ $32 \mathrm{~s}$, respectivamente.

\subsection{Caracterização do Lixiviado}

A caracterização físico-química do lixiviado foi realizada tanto para o LPT quanto para o efluente resultante da OASc. Os valores de $\mathrm{pH}$, condutividade elétrica (CE) e turbidez foram determinados por potenciometria utilizando, respectivamente, um $\mathrm{pH}$-metro Digimed DM/20, um medidor multiparâmetro Hanna HI4522 e um turbidímetro portátil Digimed DMTU, de acordo com a metodologia dos equipamentos. A cor aparente e verdadeira foi quantificada por colorimetria em espectrofotômetro visível Hach DR-2010 (Hach Company, 1996, Método 8025). A demanda química de oxigênio (DQO) foi determinada em triplicata pelo método colorimétrico (APHA/AWWA/WEF, 1998, Método 5220D) utilizando um espectrofotômetro Hach DR-2010, com amostras previamente digeridas a $150{ }^{\circ} \mathrm{C}$ em um Hach COD-Reactor 45600. O carbono orgânico total (COT) foi analisado em triplicada pelo método de combustão a alta temperatura (APHA/AWWA/WEF, 1998) utilizando um analisador de COT Shimadzu TOC-L CSH. A determinação do nitrogênio amoniacal total (N$\mathrm{NH}_{3}$ ) ocorreu por potenciometria, utilizando um eletrodo seletivo de íons amônia (Thermo Scientific Orion 9512BNWP) e um medidor multi-parâmetro Thermo Scientific Orion 4-Star, de acordo com a metodologia do fabricante.

\section{RESULTADOS E DISCUSSÃO}

Na Tabela 1 estão apresentadas as características do LPT e após a oxidação pelo processo supercrítico.

Conforme apresentado na Tabela 1, foi verificado que, mesmo após tratamento por processos físicos, químicos e biológicos, o lixiviado não se enquadrou nos parâmetros de lançamento exigidos pelas legislações ambientais, em relação às concentrações de matéria orgânica, em termos de DQO, e amônia. Esse fato é decorrente da alta complexidade desse efluente e indica a necessidade de seu tratamento adicional. De acordo com Kylefors et al. (2003), a alta DQO ocorre devido à presença de compostos inorgânicos no lixiviado, como $\mathrm{Fe}(\mathrm{II}), \mathrm{Mn}(\mathrm{II})$, sulfeto, compostos nitrogenados e íons amônia e cloretos. 


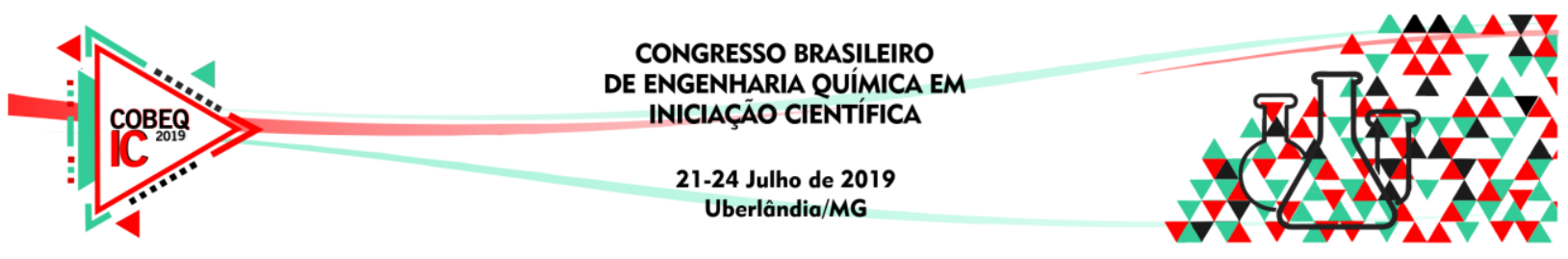

Tabela 1 - Características do lixiviado pré-tratado (LPT) e após OASc a 600 e $700{ }^{\circ} \mathrm{C}$

\begin{tabular}{|c|c|c|c|c|}
\hline Parâmetros & LPT & $\begin{array}{c}\text { Limite para } \\
\text { descarte }\end{array}$ & OASc $600^{\circ} \mathrm{C}$ & OASc $700^{\circ} \mathrm{C}$ \\
\hline $\mathrm{pH}$ & 7,8 & $5,0-9,0^{\mathrm{a}}$ & 7,3 & 6,9 \\
\hline Cor Aparente (mg Pt-Co L $\left.{ }^{-1}\right)$ & 608 & - & 27 & 21 \\
\hline Cor Verdadeira (mg Pt-Co L $\left.{ }^{-1}\right)$ & 351 & - & 16 & 20 \\
\hline Turbidez & 18,3 & - & 1,6 & 1,4 \\
\hline DQO $\left(\mathrm{mg} \mathrm{L}^{-1}\right)$ & 395 & $200^{\mathrm{b}}$ & 153 & 15 \\
\hline $\operatorname{COT}\left(\mathrm{mg} \mathrm{L}^{-1}\right)$ & 130 & - & 22 & 13 \\
\hline COT/DQO & 0,3 & - & 0,1 & 0,9 \\
\hline $\mathrm{CE}\left(\mu \mathrm{S} \mathrm{cm}^{-1}\right)$ & 8065 & - & 824 & 703 \\
\hline $\mathrm{N}-\mathrm{NH}_{3}\left(\mathrm{mg} \mathrm{L}^{-1}\right)$ & 25 & $20^{\mathrm{a}}$ & 104 & 82 \\
\hline
\end{tabular}

Notas: Estabelecido pelas Resoluções (a) CONAMA no 430 (Brasil, 2011) e (b) CEMA nº 070 (Paraná, 2009).

O processo de OASc a $600{ }^{\circ} \mathrm{C}$ foi eficiente para a degradação do lixiviado, promovendo altas reduções dos parâmetros de cor aparente (96\%) e verdadeira (95\%), turbidez (91\%), COT (83\%) e DQO (61\%). Uma vez que a cor e a turbidez estão relacionadas com as concentrações de materiais dissolvidos e suspensos, respectivamente, e a CE com os sais do efluente, há a indicação de que estes compostos também foram removidos pela OASc. Por outro lado, o N-NH$H_{3}$ teve a sua concentração aumentada após a OASc a $600{ }^{\circ} \mathrm{C}$. Isso indica que os compostos nitrogenados presentes no lixiviado foram apenas parcialmente oxidados, formando a amônia que é subproduto recalcitrante intermediário nesse processo de oxidação.

A menor redução de DQO pela OASc a $600{ }^{\circ} \mathrm{C}$ indicou que ainda estavam presentes no lixiviado significativas concentrações de compostos inorgânicos e orgânicos nitrogenados, os quais também são oxidados na técnica analítica utilizada para o dimensionamento da DQO. Devido a isso, a remoção de material orgânico em processos de tratamento tem sido avaliada pela relação COT/DQO. Após elevação da OASc para $700{ }^{\circ} \mathrm{C}$, essa relação se elevou, indicando que os compostos ainda presentes no lixiviado eram de natureza orgânica, provenientes de compostos carbonáceos.

Ao elevar a temperatura do sistema supercrítico para $700{ }^{\circ} \mathrm{C}$, foi verificado pouco incremento na redução dos parâmetros avaliados, com exceção da matéria orgânica, representada pela DQO e pelo COT, os quais alcançaram remoções de $96 \%$ e 90\%, respectivamente, em relação à concentração inicial do LPT. Além disso, o aumento da temperatura de OASc permitiu a degradação da amônia formada no processo, o que corrobora com a afirmação de Zou et al. (2014), os quais citam que, para uma completa degradação de compostos nitrogenados, são necessárias elevadas temperaturas e/ou tempo de reação. Nesse sentido, devido à complexidade do lixiviado, ainda seria necessário aumentar esses parâmetros para se alcançar uma qualidade no nível de lançamento em corpos hídricos.

\section{CONCLUSÃO}

O processo de OASc, em ambas as temperaturas, 600 e $700{ }^{\circ} \mathrm{C}$, foi eficiente na degradação do lixiviado pré-tratado por processos convencionais no próprio aterro, com exceção do $\mathrm{N}^{-\mathrm{NH}_{3}}$. $\mathrm{O}$ aumento de temperatura favoreceu a oxidação de compostos 


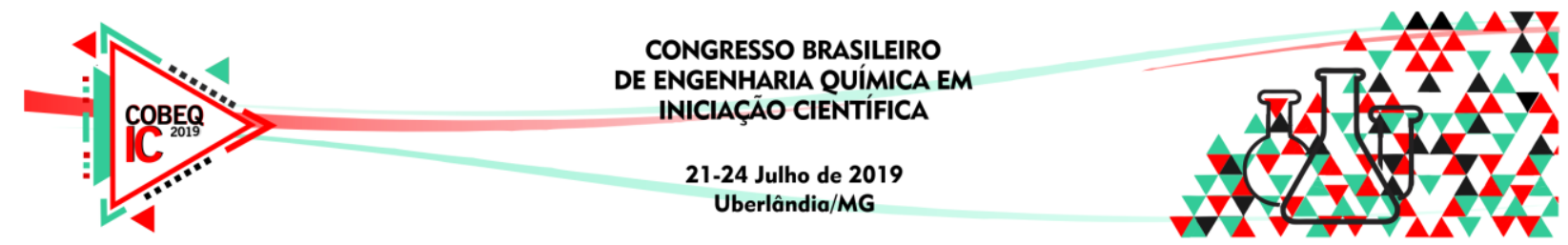

nitrogenados; entretanto não foi suficiente para a sua completa transformação a $\mathrm{N}_{2}$. Dessa forma, condições ainda mais severas de operação do processo de OASc seriam necessárias para promover a completa redução desse composto recalcitrante e complexo. Alternativamente, o mesmo poderia ser removido por adsorção, utilizando materiais adsorventes específicos, como zeólitas.

\section{REFERÊNCIAS}

APHA (American Public Health Association), AWWA (American Water Works Association), WEF (Water Environment Federation). Standard methods for the examination of water and wastewater. 20th ed. USA: APHA, 1998.

BRASIL. Ministério do Meio Ambiente. CONAMA (Conselho Nacional de Meio Ambiente). Resolução $n^{\circ} 430$, de 13 de maio de 2011.

HACH COMPANY. Procedures Manual of Spectrophotometer DR/2010. USA: Hach Company, 1996.

KJELDSEN, P. BARLAZ, M. A.; ROOKER, A. P.; BAUN, A.; LEDIN, A.; CHRISTENSEN, T. H. Present and long-term composition of MSW landfill leachate: a review. Crit. Rev. Environ. Sci. Technol., v. 32, p. 297-336, 2002.

KYLEFORS, K.; ECKE, H.; LAGERKVIST, A. Accuracy of COD test for landfill leachates. Water Air Soil Pollut., v. 146, p. 153-169, 2003.

NATIONAL INSTITUTE OF STANDARDS AND TECHNOLOGY (NIST). NIST Standard Reference Database 69: NIST Chemistry WebBook. U.S. Secr. Commer. behalf United States Am. URL https://webbook.nist.gov/chemistry/>. Acesso em 13 jul. 2018.

PARANÁ. CEMA (Conselho Estadual de Meio Ambiente). Secretaria de Estado do Meio Ambiente e Recursos Hídricos - SEMA. Resolução $n^{\circ}$ 070, de 01 de outubro de 2009.

SCANDELAI, A. P. J.; CARDOZO FILHO, L.; MARTINS, D. C. C.; FREITAS, T. K. F. S.; GARCIA, J. C.; TAVARES, C. R. G. Combined processes of ozonation and supercritical water oxidation for landfill leachate degradation. Waste Manage., v. 77, p. 466-476, 2018.

SCANDELAI, A. P. J.; F. B. MORO; M. G. LINHARES; MARTINS, D. C. C.; CARDOZO FILHO, L.; TAVARES, C. R. G. Oxidação em água supercrítica como tratamento complementar de lixiviado de aterro sanitário. In: Simpósio de Engenharia Química, VII, 2017, Maringá. Anais... Maringá: UEM, 2017.

VAN BENNEKOM, J. G.; VENDERBOSCH, R. H.; ASSINK, D.; HEERES, H. J. Reforming of methanol and glycerol in supercritical water. J. Supercrit Fluids, v. 58, p. 99-113, 2011.

WEIJIN, G.; XUEJUN, D. Degradation of landfill leachate using transpiring-wall supercritical water oxidation (SCWO) reactor. Waste Manage., v. 30, p. 2103-2107, 2010.

ZOU, D.; CHI, Y.; DONG, J.; FU, C.; NI, M. Supercritical water oxidation of MSW leachate: factor analysis and behavior of heavy metals. Environ. Prog. Sustain. Energy, v. 33, n. 4, p. 1117-1124, 2014. 